\title{
Retrosigmoid Approach for Trapping and Removal of a Distal Dissecting Superior Cerebellar Artery Aneurysm in a Child
}

\author{
Javier Ros de San Pedro ${ }^{1}$ \\ ${ }^{1}$ Regional Service of Neurosurgery, Vascular Neurosurgery Unit, \\ Hospital Clínico Universitario Virgen de la Arrixaca, El Palmar \\ (Murcia), Spain \\ J Neurol Surg B 2019;80(suppl S3):S335-S338.
}

\begin{abstract}
Keywords

- cerebellopontine angle

- dissecting aneurysm

- distal superior cerebellar artery

- aneurysm trapping

- retrosigmoid craniotomy

Objectives To demonstrate the feasibility of the retrosigmoid craniotomy for surgical management of vascular lesions located in the cerebellopontine angle (CPA).

Method A previously healthy 2-year-old boy presented a sudden episode of torticollis to the left while sleeping. This episode was selflimited but it occurred two more times in a 6day span. Torticollis worsened in the upright position, caused unsteady gait and refusal to walk from the child. The preoperative magnetic resonance imaging (MRI) showed the presence of a round, heterogenous vascular lesion in the left CPA. The lesion clearly enhanced after contrast administration. The preoperative angiography demonstrated the absence of left anterior inferior cerebellar artery anterior inferior cerebellar artery (AICA), being the left superior cerebellar artery (SCA) the supplier of the left lateral cerebellum. A blurred blush on the distal left SCA was compatible with a fusiform aneurysm. A standard retrosigmoid approach was planned for trapping and removal of the aneurysm.

Results Through a left retrosigmoid craniotomy the aneurysm was approached, along with the different neurovascular structures of the CPA. The aneurysm leaned on the VII, VIII nerves complex and the superior petrosal vein, while tightly attached to the lateral cerebellum. Both proximal and distal SCA segments to the aneurysm were dissected, clipped, and divided for a complete trapping. Finally, the aneurysm was completely detached and removed in a whole piece. The patient fully recovered after surgery with no relapse of his symptoms.

Conclusion The retrosigmoid craniotomy is a versatile approach that permits wide exposure of all CPA structures and adequate removal of distal aneurysms located in those cerebellar arteries supplying the lateral cerebellum.

The link to the video can be found at: https://youtu.be/oEVfy4goFYM.
\end{abstract}

Conflict of Interest

None.

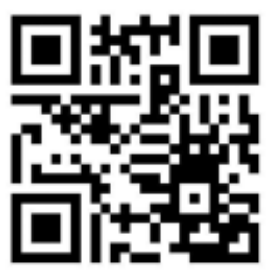

www.thieme.com/skullbasevideos

www.thieme.com/jnlsbvideos

received

May 14, 2018

accepted

August 19, 2018

published online

October 15, 2018
DOI https://doi.org/

10.1055/s-0038-1675172.

ISSN 2193-6331.
๑) 2019 Georg Thieme Verlag KG
Stuttgart · New York

License terms

(c) (1) $\ominus$ (\$) 

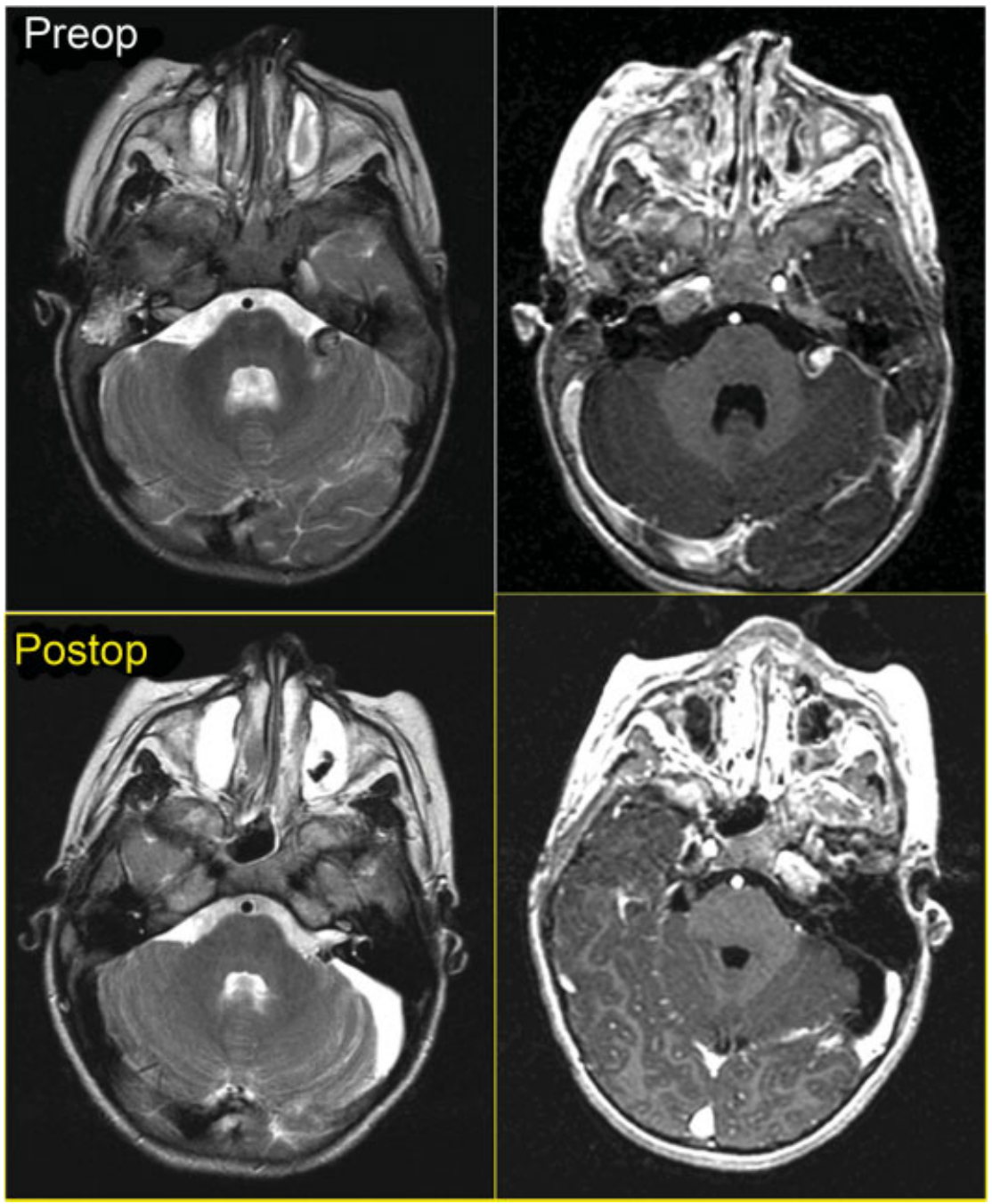

Fig. 1 Comparison between pre- and postoperative MRI. The upper left image corresponds to an axial T2WI slice, where the SCA aneurysm is displayed as a heterogenous, round lesion in the left CPA, which causes slight edema on the neighboring middle cerebellar peduncle. The lower left image is an axial postoperative T2WI, which shows the left CPA with no relapsing aneurysm. Note that the perilesional edema in the middle cerebellar peduncle has completely disappeared. The upper right image corresponds to an axial T1WI after intravenous contrast administration, which clearly depicts the dissecting SCA aneurysm along with the proximal and distal parent artery. The lower right image is an axial postoperative T1WI after contrast injection that demonstrates the absence of the aneurysm, whereas the patent SCA is distinctly shown. CPA, cerebellopontine angle; MRI, magnetic resonance imaging; Postop, postoperative; Preop, preoperative;SCA, superior cerebellar artery; WI, weighted image. 


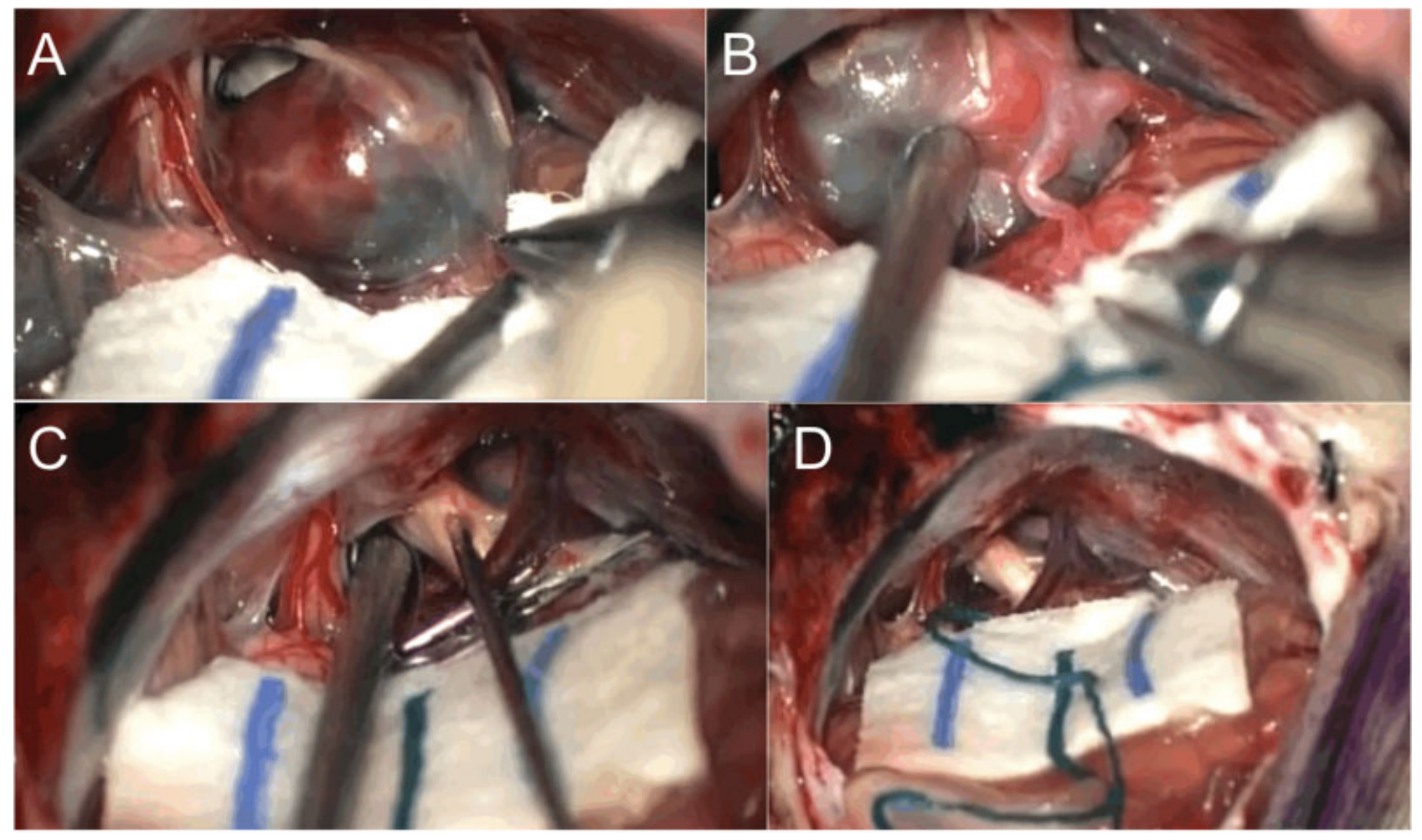

Fig. 2 Intraoperative images through a retrosigmoid craniotomy. (A) Distal dissecting SCA aneurysm in the left CPA. The aneurysm is tightly adhered to the cerebellum on its posterior and medial aspects, whereas its inferior pole leans on the VII, VIII nerves complex. (B) The aneurysm neck is completely shown, including the proximal and distal parent artery segments. Due to the neck shape this aneurysm was deemed unclippable, for which trapping and removal was chosen as the best surgical option. (C) Complete left CPA exposure after aneurysm removal. Note the presence of two aneurysm clips. The superior petrosal vein, trigeminal nerve, and the VII-VIII nerves complex are clearly shown. A stimulator probe is applied on the $V$ nerve checking its function. (D) Final view of the left CPA after aneurysm removal. The same structures as in $\mathrm{C}$ are shown, plus the lower cranial nerves caudally to the facial-acoustic complex. CPA, cerebellopontine angle; SCA, superior cerebellar artery. 